\title{
GEOLOGICAL SURVEY OF FINLAND RADIOCARBON MEASUREMENTS IV
}

E. HYYPPÄ, A. HEIKKINEN, and V. TOIVONEN

$\mathrm{C}^{1+}$ Laboratory, Geological Survey of Finland, Otaniemi, Finland

The following results represent measurements carried out since our 3rd date list was prepared. The entire $\mathrm{CO}_{2}$ purification technique (Radiocarbon, 1962, v. 4, p. 81-83) has been changed to "wet purification." No measurements of $\mathrm{C}^{12} / \mathrm{C}^{13}$ ratios have been made.

\section{GEOLOGIC SAMPLES}

\section{Su-34. Kuusamo, E Finnish Lapland}

$$
7430 \pm 190
$$

Brvales peat taken with piston samplo

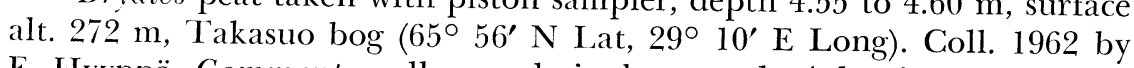
E. Hyyppä. Comment: pollen analysis shows early Atlantic period.

\section{Su-35. Kuusamo, E Finnish Lapland}

$9320 \pm 170$

Same bog as Su-34, fine detritus gyttja taken with piston sampler, depth 4.80 to $4.85 \mathrm{~m}$. Coll. 1962 by E. Hyyppä. Comment: pollen analysis shows later part of Betula maximum time. The C-14 date is possibly too old for this horizon in Lapland.

\section{Su-36. Pello, W Finnish Lapland}

$8250 \pm 150$

Fine detritus gyttja taken with piston sampler, depth 5.53 to $5.65 \mathrm{~m}$, surface alt. $101.4 \mathrm{~m}$, Kaakkuririipi bog (66 $47^{\prime} \mathrm{N}$ Lat, $24^{\circ} 15^{\prime} \mathrm{E}$ Long). Coll. 1962 by E. Hyyppä. Comment: pollen and diatom analysis indicate early Littorina I stage at beginning of Atlantic period (Hyyppä, 1966).

\section{Su-37. Virolahti, SE Finland}

$7835 \pm 170$

Allochtonous peat and coarse detritus gyttja taken with piston sampler, depth 5.05 to $5.12 \mathrm{~m}$, surface alt. $27.11 \mathrm{~m}$, Ruokolampi bog $\left(60^{\circ}\right.$ $32^{\prime} \mathrm{N}$ Lat, $27^{\circ} 40^{\prime} \mathrm{E}$ Long). Coll. 1961 by M. Salmi. Comment: pollen and diatom analysis indicate beginning of Littorina I stage (Salmi, 1961).

\section{Su-38. Sippola, SE Finland}

$9710 \pm 230$

Sphagnum-Carex-Equisetum peat taken with piston sampler, depth 6.55 to $6.65 \mathrm{~m}$, surface alt. $52.42 \mathrm{~m}$, Hangassuo bog $\left(60^{\circ} 47^{\prime} \mathrm{N}\right.$ Lat, $26^{\circ}$ 55’ E Long). Coll. 1948 by M. Salmi. Comment: pollen analysis shows Pre-Boreal Betula maximum (Salmi, 1948).

\section{Su-39. Kuusamo, E Finnish Lapland}

$8080 \pm 150$

Coarse detritus gyttja from drowned peat bog, taken with piston sampler, depth 3.03 to $3.08 \mathrm{~m}$, surface alt. $251.06 \mathrm{~m}$, Joukamojärvi $\left(65^{\circ}\right.$ 
52' N Lat, 30 E Long). Coll. 1963 by E. Hyyppä. Comment: pollen analysis shows Betula maximum in beginning of Atlantic period.

\section{Su-40. Pello, W Finnish Lapland}

$8030 \pm 200$

Terrestrial peat taken with piston sampler, depth 6.0 to $6.1 \mathrm{~m}$, surface alt. $228.76 \mathrm{~m}$, Tornio R. Valley $\left(66^{\circ} 46^{\prime} \mathrm{N}\right.$ Lat, $24^{\circ} 04^{\prime} \mathrm{E}$ Long). Coll. 1961 by E. Hyyppä. Sample coll. from same profile as Su-24 (Radiocarbon, 1963, v. 5, p. 303) in which surface alt. $288 \mathrm{~m}$ was in error. Comment: sample lay directly above Betula wood dated 6200 B.c.

Su-42. Luumäki, SE Finland

Modern

Wood under sand layer, Käännetsalo Is. $\left(60^{\circ} 55^{\prime} \mathrm{N}\right.$ Lat, $27^{\circ} 30^{\prime} \mathrm{E}$ Long). Coll. 1965 by E. Hyyppä. Comment: wood belongs to very late transgression of Lake Saimaa.

\section{Su-43. Virolahti, SE Finland}

$8660 \pm 240$

6710 в.c.

Fine fresh-water mud taken with piston sampler, depth 7.70 to $7.80 \mathrm{~m}$, surface alt. $36.0 \mathrm{~m}$, Huosiosuo bog $\left(60^{\circ} 33^{\prime} \mathrm{N}\right.$ Lat, $27^{\circ} 38^{\prime} \mathrm{E}$ Long). Coll. 1966 by E. Hyyppä. Comment: pollen and diatom analysis indicate Pre-Boreal regression maximum from Yoldia II to Yoldia IV; horizon represents isolation and is overlain by thin layer belonging to Yoldia IV transgression (Hyyppä, 1966).

\section{Su-44. Enontekiö, NW Finnish Lapland}

$2560 \pm 100$

Sorbus aucuparia wood from layer of fine sand and sand, taken with piston sampler, depth 2.5 to $3.0 \mathrm{~m}$, Markkina $\left(68^{\circ} 30^{\prime} \mathrm{N}\right.$ Lat, $22^{\circ} 20^{\prime} \mathrm{E}$ Long). Coll. 1966 by H. Roivainen. Comment: horizon represents beginning of Sub-Atlantic flood period.

\section{Su-45. Nurmo, W Finland}

$4285 \pm 130$

2335 в.c.

Sphagnum-Carex peat from hand-dug sec., depth 1.36 to $1.39 \mathrm{~m}$, surface alt. $93.9 \mathrm{~m}$, Kurjenneva bog (62 $46^{\prime} \mathrm{N}$ Lat, $23^{\circ} 08^{\prime} \mathrm{E}$ Long). Coll. 1962 by M. Salmi. Comment: pollen analysis shows beginning of Picea curve (Salmi, 1963).

\section{Su-46. Nurmijärvi, S Finland}

$6420 \pm 130$

Carex-Sphagnum peat taken with piston sampler, depth 0.4 to $0.5 \mathrm{~m}$, surface alt. $69 \mathrm{~m}$, Råbacka bog (60 $29^{\prime} \mathrm{N}$ Lat, 24 57' E Long). Coll. 1966 by K. Virkkala. Comment: sample lay directly under horizon showing beginning of pollen-analytical Picea curve (Virkkala, 1966).

\section{Su-47. Joensuu, E Finland}

$\mathbf{5 9 5 0} \pm \mathbf{1 5 0}$

Betula wood from sand layer, taken with piston sampler, depth

4000 в.c. $3.15 \mathrm{~m}$, surface alt. $84.68 \mathrm{~m}$, Raatekangas (62 $40^{\prime} \mathrm{N}$ Lat, $29^{\circ} 50^{\prime} \mathrm{E}$ Long). 
Coll. 1967 by E. Hyyppä. Comment: wood belongs to early transgression of Lake Saimaa.

\section{Su-48. Hauho, S Finland}

$$
1250 \pm 70
$$

Wood from layer of sand, depth $0.3 \mathrm{~m}\left(61^{\circ} 15^{\prime} \mathrm{N}\right.$ Lat, 700 A.D. Long). Coll. 1966 by E Hyppä. Commen $15^{\prime} \mathrm{N}$ Lat, $24^{\circ} 40^{\prime} \mathrm{E}$ late transgression of Lake Vanajavesi.

\section{Su-49. Imatra, SE Finland}

$\mathbf{8 2 5 0} \pm \mathbf{2 0 0}$

Bryales peat containing remnants of deciduous trees from under silty mud, depth $11.0 \mathrm{~m}$, surface alt. $75.65 \mathrm{~m}$, Kaukopää $\left(61^{\circ} 10^{\prime} \mathrm{N}\right.$ Lat, $28^{\circ} 50^{\prime}$ E Long). Coll. 1966 by E. Hyyppä. Comment: pollen analysis shows latter half of Boreal period; horizon antedates transgression of
Lake Saimaa.

\section{Su-50. Joensuu, E Finland}

$5810 \pm 150$

Sandy peat from under fine sand, Surakka $\left(62^{\circ} 40^{\prime} \mathrm{N}\right.$ Lat, $29^{\circ} 40^{\prime} \mathrm{E}$ Long). Coll. 1960 by E. Hyyppä. Comment: sample represents early stage of Lake Saimaa transgression.

\section{Su-51. Asikkala, S Finland}

$7550 \pm 180$

Pinus wood from under Lat, $25^{\circ} 30^{\prime} \mathrm{F}$ Long) Lat, $25^{\circ} 30^{\prime}$ E Long). Coll. 1963 by H. Peltola and E. Hyyppä. Comment: wood indicates forest drowned by early Lake Päijänne transgression.

\section{Su-52. Helsinki, S Finland}

$4940 \pm 140$ 2990 B.c.

Alnus wood from hand-dug sec., depth $2.80 \mathrm{~m}$, surface alt. ca. $30 \mathrm{~m}$, Myllypuro $\left(60^{\circ} 14^{\prime} \mathrm{N}\right.$ Lat, $25^{\circ} 07^{\prime} \mathrm{E}$ Long). Coll. 1965 by E. Hyyppä. Comment: horizon represents approx. time of Littorina IV, local shore
alt. $23 \mathrm{~m}$.

\section{Su-54. Luumäki, SE Finland}

$$
1130 \pm 100
$$

Carex peat under sand layer, alt. $75.6 \mathrm{~m}$, Käännetsalo Is. $\left(60^{\circ} 55^{\prime} \mathrm{N}\right.$ Lat, $27^{\circ} 30^{\prime} \mathrm{E}$ Long). Coll. 1965 by E. Hyyppä. Comment: peat belongs to late transgression of Lake Saimaa.

\section{Porvoo series}

Surface and threshold alt. $28.5 \mathrm{~m}$ of the Bastuberg peat bog $\left(60^{\circ} 21^{\prime}\right.$ N Lat, $25^{\circ} 47^{\prime}$ E Long). Su-41 and Su-53 coll. 1966, others 1967 by E. Hyуррӓ.

\section{Su-41.}

$6870 \pm 165$

Coarse detritus gyttja, depth 1.75 to $1.82 \mathrm{~m}$. Comment: pollen and diatom analysis indicate beginning of Littorina II stage. 
Su-53.

Coarse detritus gyttja, depth 1.27 to $1.33 \mathrm{~m}$. Comment: horizon represents time when basin had already isolated from Littorina II stage and Littorina III stage (local shore alt. $26 \mathrm{~m}$ ) was established.

$4110 \pm 130$

Su-55.

2160 B.C.

Betula bark, depth $0.65 \mathrm{~m}$. Comment: pollen analysis shows beginning of Picea curve.

Su-56.

$3910 \pm 180$

Peat containing remnants of deciduous trees, depth $0.60 \mathrm{~m}$. Comment: same as Su-55.

$\mathbf{5 8 6 0}+\mathbf{2 0 0}$

Su-57.

3910 B.c. Su-53.

Coarse detritus gyttja, depth 1.30 to $1.35 \mathrm{~m}$. Comment: same as

\section{Su-58.}

$5570 \pm 200$

Phragmites leaves and cones, depth $1.30 \mathrm{~m}$. Comment: same as Su53 and $\mathrm{Su}-57$.

\section{Su-59.}

$6010 \pm 105$

Phragmites leaves and cones, depth $1.60 \mathrm{~m}$. Comment: pollen and diatom analysis indicate maximum of Littorina II stage.

\section{Su-60.}

$7950 \pm 190$

Wood, depth $2.60 \mathrm{~m}$. Comment: horizon represents Littorina I stage directly after maximum stage.

Su-61.

$\mathbf{8 2 0 0} \pm \mathbf{2 7 0}$

6250 B.C.

Pinus wood, depth $2.80 \mathrm{~m}$. Comment: horizon represents beginning of maximum transgression of Littorina I stage.

\section{Su-62.}

$6810 \pm 165$

Wood, depth $1.80 \mathrm{~m}$. Comment: horizon represents beginning of maximum transgression of Littorina II stage.

\section{Su-63.}

$7100 \pm 170$

Wood and Phragmites leaves, depth 1.70 to $1.75 \mathrm{~m}$. Comment: horizon represents beginning of maximum transgression of Littorina II stage. 
Su-64. Helsinki, S Finland

$5110 \pm 115$

detritus gyttja, depth $3 \mathrm{~m}$. Coll. 1965 by ple belongs to final phase of Littorina III stage.

\section{Su-65. Virolahti, SE Finland}

$6310 \pm 130$ Coll. to Littorina II maximum.

Su-66. Ulvila, W Finland

Modern

Branch of Betula from sand pit, depth $15 \mathrm{~m}\left(61^{\circ} 24^{\prime} \mathrm{N}\right.$ Lat, $21^{\circ} 58^{\prime}$ E Long). Coll. 1967 by K. Virkkala. Comment: sample is from slumped portion in pit.

\section{ARCHAFOLOGIC SAMPIE}

\section{Su-67. Sodankylä, Finnish Lapland}

Modern

Pinus wood from under boat of ancient Lapp dwelling place, Sompiojärvi $\left(68^{\circ} 05^{\prime} \mathrm{N}\right.$ Lat, $27^{\circ} 27^{\prime} \mathrm{E}$ Long). Coll. 1964 by E. Hyyppä.

Date lists:

\section{REFERENCFS}

Finland I Hyyppä, Hoffrén, and Isola, 1962

Finland II Hyyppä, Isola, and Hoffrén, 1963

Finland III Hyyppä, Toivonen, and Isola, 1964

Hyyppä, E., 1966, The late-Quaternary land uplift in the Baltic sphere and the relation diagram of the raised and tilted shore levels: Acad. Sci. Fennicae. Annales, A, III, 90 , p. 153-168.

Hyyppä, E., Hoffrén, V., and Isola, A., 1962, Geological Survey of Finland radiocarbon measurements I: Radiocarbon, v. 4, p. 81-83.

Hyyppä, E., Isola, A., and Hoffrén, V., 1963, Geological Survey of Finland radiocarbon measurements II: Radiocarbon, v, 5, p. 302-304.

Hyyppä, E., Toivonen, A. V. P., and Isola, A., 1964, Geological Survey of Finland radiocarbon measurements III: Radiocarbon, v. 6, p. 110-111.

Salmi, Martti, 1948, Die Ancylustransgression in dem Moore Hangassuo im Süd-Finn land: Finland, Comm. Géol. B. no. 142, p. 1-20. 1961, Two Littorina transgression in Virolahti, southeastern Finland; Finland, Comm. Géol. B. no. 196, p. 417-436.

1963, Drei subfossile Sattelrobben aus Ostbottnien; geologische Datierung der Funde und einige chronologische Beobachtungen: Arch. Soc. "Vanamo", v. $18: 2$, p. $82-95$.

Virkkala, K., 1966, Radiocarbon ages of the Råbacka bog, southern Finland: Finland, Comm. Géol. B. no. 222, p. 237-240. 